\title{
The identification of concepts defined by attribute change
}

\author{
ROBERT C. HAYGOOD \\ Arizona State University, Tempe, Arizona 85281 \\ and \\ HERBERT H. BELL \\ University of Tennessee at Nashville, Nashville, Tennessee 37203
}

\begin{abstract}
A new procedure for concept learning is described, in which concepts are defined by change in relevant stimulus dimensions rather than by the standard method which uses fixed levels of dimensions. Two experiments are reported. The first shows that change concepts are more difficult to learn than fixed-level concepts. The second experiment shows the relative difficulty of conjunctive and disjunctive concepts is reversed in change problems. Possible explanations for these effects are discussed.
\end{abstract}

Experimental studies of concept identification have almost universally used concepts defined by the presence or absence of specific stimulus attributes (Bourne, 1966). For example, the conjunctive concept "red and square" specifies that the attributes red and square must be present in all examples of the concept. Similarly, a concept based on the logical rule of inclusive disjunction might be that all examples are "either red or square or both." The attributes have typically, though not always, represented levels or values on well defined stimulus dimensions such as hue, size, number, horizontal and vertical position, and so forth.

It is clear that there are numerous concepts in daily life that are based, not on fixed levels of stimulus dimensions, but on dimensional change. The concept of approach, for example, is defined by a change in horizontal position; similar examples involving changes in hue, size, and the like are easy to imagine. Despite the seeming commonness of concepts based on stimulus change (or on its complement, retention of attributes on successive stimuli), they have received little, if any, experimental attention. Thus, essentially nothing is known about the factors determining the ease of identifying the relevant change in a stimulus series.

It is quite easy to construct an attribute-change problem as a counterpart to an ordinary fixed attribute concept-identification problem. Instead of selecting one or more specific relevant attributes, one selects dimensions along which the relevant changes are to occur. To take a simple example, the change counterpart of the conjunctive concept "red and square" would be "changed in both hue and shape." Both of these problems would normally use the same stimulus

This research was supported in part by a grant ( $\mathrm{MH} \mathrm{11283}$ ) from the National Institute of Mental Health, United States Public Health Service. Requests for reprints should be addressed , Haygood, Department of Psychology, Arizona State Unversity, Tempe, Arizona 85281. patterns, and only the system of reinforcement would determine which concept is learned.

The purposes of the present study were (a) to demonstrate the feasibility of studying attribute-change concepts with the standard concept-identification methodology, and (b) to gather some preliminary evidence on the effect of conceptual rule in both types of problems.

\section{EXPERIMENT I}

The purpose of this experiment was to compare the learning of simple one-dimensional fixed and change concepts. In addition, it was designed to serve as pretraining for Experiment II, which followed immediately in the same experimental session.

The task and procedure were much like those used in previous experiments (e.g., Haygood \& Bourne, 1965). The subject's task was to learn the correct concept by being shown stimulus patterns, selecting one of two possible classification responses, and receiving corrective feedback. The stimuli were geometric designs which varied along five binary dimensions, hue (red or blue), shape (triangle or square), size (large or small), number (one or two figures in a pattern), and background (plain or striped). The concept to be learned consisted of either a single fixed relevant attribute (small or triangle) or a single relevant change dimension (size or shape), and each problem had four irrelevant dimensions. The experimental design thus was a 2 by 2 factorial, with two types of concepts and two choices of relevant stimulus dimension.

The subjects were 48 students from introductory psychology classes at Kansas State University. They were assigned to the four treatment combinations in order of appearance, in such a way that the groups were filled at the same rate. All subjects were naive in that they had 
Table 1

Error Performance During Experiment II

\begin{tabular}{lccccc}
\hline & \multicolumn{3}{c}{ Conceptual } & Rule \\
\cline { 2 - 3 } & \multicolumn{2}{c}{ Conjunction } & & \multicolumn{2}{c}{$\begin{array}{c}\text { Inclusive } \\
\text { Disjunction }\end{array}$} \\
\cline { 2 - 3 } \cline { 5 - 6 } Type of Problem & Mean & SD & & Mean & SD \\
\hline Fixed Attribute & 2.33 & 1.50 & & 4.83 & 3.54 \\
Attribute Change & 11.16 & 9.42 & & 5.75 & 6.64 \\
\hline
\end{tabular}

not previously participated in a concept-learning experiment.

Detailed instructions were given at the outset which described the nature of the concept (fixed or change), the stimulus population, the method of responding, and the meaning of the feedback signal lamps. After the instructions were given, the patterns were rear-projected, one at a time, on a screen directly in front of the subject. The subject responded by pressing one of two buttons labeled YES and NO for the fixed problems, or CHANGE and NO CHANGE for the change problems. Following each response, the subject was given feedback by illuminating a signal lamp directly above the correct button. The subject was given as much time as needed to respond, and instructions stressed accuracy rather than speed. The criterion of solution for all problems was 15 consecutive correct responses, and all subjects were able to solve the problem. However, four subjects were subsequently replaced for failure to follow instructions in Experiment II.

The results showed that the change problem was considerably more difficult than the fixed problem, with mean errors of 26.6 and 12.1 , respectively. This was the only significant effect in the analysis of variance of errors $[\mathrm{F}(1,44)=4.99, \mathrm{p}<.05]$.

\section{EXPERIMENT II}

Experiment II was designed to compare the learning of fixed and change problems constructed with two different conceptual rules, conjunction and inclusive disjunction. The fixed conjunctive concept was "red and square," matched by the corresponding change conjunction, "change in both color and shape." Similarly, the fixed inclusive disjunction was "either red or square, or both" with the corresponding change concept "change in either hue or shape or both." These combinations created a 2 by 2 by 2 factorial design with two types of concept, two conceptual rules, and the additional factor of whether the type of concept was the same as in Experiment I or different. Each problem had two relevant and one irrelevant (size) dimensions.

The procedure was essentially the same as that of Experiment I. Immediately following Experiment I, each subject was given further instructions concerning Experiment II. Three of the 12 subjects in each
Experiment I condition were transferred to each of the four conditions in Experiment II to insure equivalent pretraining experience in all groups. For half of each group, this mean a change to a different type of concept, and the new type was thoroughly explained to each subject. After these instructions were given, the problem was begun. As previously noted, four subjects failed to follow instructions properly, and were replaced.

The results showed that, as in Experiment I, performance on the fixed problem was superior to that on the change problem $[F(1,40)=7.31, p<.01]$. There was no significant difference between conceptual rules overall, but the interaction of rule and type of concept was significant $[\mathrm{F}(1,40=4.69, \mathrm{p}<.05]$. This interaction is shown clearly in Table 1 . The fixed problem follows previous results (e.g., Haygood \& Bourne, 1965) as expected, with conjunction easier than inclusive disjunction. The relationship is reversed, however for the change problem, with disjunction easier. There was no significant difference between the subjects who had the same type of concept in both experiments and those who changed $(F=0.18)$, and neither of the interactions of this variable with the type of concept or the rule used during Experiment II was significant.

\section{GENERAL DISCUSSION}

The results indicate clearly that the attribute-change procedure generates a practical experimental problem, and that the subjects are capable of solving such problems without unreasonable difficulty. That this procedure may prove useful in studies of conceptual behavior is suggested by the unexpected reversal of difficulty of conjunctive and inclusive disjunctive concepts in the change problem.

The greater difficulty of solving change problems probably stems from the greater memory requirements. Correct responding in the change problem requires that the subject retain attributes from the previous stimulus pattern and compare them with the attributes in the present stimulus. In contrast, the fixed problem requires only that the subject examine the present stimulus. The alternative possibility that the differences were caused by greater familiarity with fixed attribute classification procedures is weakened by the absence of differences (in Experiment II) attributable to Experiment I pretraining.

The reversal of order of difficulty for conjunction and inclusive disjunction in the change problem probably reflects a peculiarity in the nature of disjunctive change. When change in either one or both attributes generates a positive instance, the negative category is defined by the conjunctive complement "both attributes the same." Presumably, the subjects were able to take advantage of the existence of this easier solution, with the result that the problem labeled "disjunctive" was actually solved as a conjunction on the negative category. A similar effect with fixed concepts was reported by Neisser and Weene (1962), who found that the extreme difficulty of conditional concepts vanished as subjects gained in sophistication, apparently because the use of two-level stimulus dimensions allowed solution of a conditional problem as a conjunction on the negative category.

The conjunctive change problem described here can also be characterized as a relational concept (cf. Bruner, Goodnow, \& Austin, 1956) of the inequality type; for any pair of successive 
stimulus patterns, the second is a positive instance if the levels of the relevant stimulus dimensions are all different. Conversion of the change problem to a standard relational concept-learning problem would require only that two patterns be presented simultaneously. Pilot studies exploring such a procedure suggest that the outcomes are much like those reported here.

\section{REFERENCES}

Bourne, L. E., Jr. Human conceptual behavior. Boston: Allyn \&
Bacon, 1966.

Bruner, J. S., Goodnow, J. J., \& Austin, G. A. A study of thinking. New York: Wiley, 1956.

Haygood, R. C., \& Bourne, L. E. Jr. Attribute- and rule-learning aspects of conceptual behavior. Psychological Review, 1965, 72, 175-195.

Neisser, U., \& Weene, P. Hierarchies in concept attainment. Journal of Experimental Psychology, 1962, 64, 640-645.

(Received for publication January $16,1975$. ) 